\title{
Erratum to: A hierarchical multi-criteria sorting approach for recommender systems
}

\author{
Luis Del Vasto-Terrientes ${ }^{1}$ - Aida Valls ${ }^{1}$. \\ Piotr Zielniewicz ${ }^{2}$. Joan Borràs ${ }^{3}$
}

Published online: 4 September 2015

(C) Springer Science+Business Media New York 2015

\section{Erratum to: J Intell Inf Syst \\ DOI 10.1007/s10844-015-0362-7}

Equation 6 of the original version unfortunately contained a mistake. The second condition on Equation 6 says

$$
\text { if } \Phi_{j}(a)-\Phi_{j}\left(b_{h}^{j}\right) \geq v_{j}\left(b_{h}^{j}\right)
$$

must be

$$
\text { if } \Phi_{j}(a)-\Phi_{j}\left(b_{h}^{j}\right) \geq p_{j}\left(b_{h}^{j}\right)
$$

i.e., " $v$ " must be replace by " $p$ ". The correct equation is shown next page:

The online version of the original article can be found at http://dx.doi.org/10.1007/s10844-015-0362-7.

Luis Del Vasto-Terrientes

luismiguel.delvasto@urv.cat

Aida Valls

aida.valls@urv.cat

Piotr Zielniewicz

piotr.zielniewicz@cs.put.poznan.pl

Joan Borràs

joan.borras@pct-turisme.cat

1 Departament d'Enginyeria Informàtica i Matemàtiques, Universitat Rovira i Virgili, Av Països Catalans, 2643007 Tarragona, Catalonia Spain

2 Institute of Computing Science, Poznan University of Technology, Piotrowo 2, 60-965 Poznan Poland

3 Parc Científic Tecnològic de Turisme i Oci, C/ Joanot Martorell, 1543480 Vila-seca, Catalonia Spain 


$$
c_{j}\left(a, b_{h}^{j}\right)= \begin{cases}1 & \text { if } \Phi_{j}(a)-\Phi_{j}\left(b_{h}^{j}\right) \leq q_{j}\left(b_{h}^{j}\right), \\ 0 & \text { if } \Phi_{j}(a)-\Phi_{j}\left(b_{h}^{j}\right) \geq p_{j}\left(b_{h}^{j}\right), \\ \frac{p_{j}\left(b_{h}^{j}\right)-\left(\Phi_{j}(a)-\Phi_{j}\left(b_{h}^{j}\right)\right)}{p_{j}\left(b_{h}^{j}\right)-q_{j}\left(b_{h}^{j}\right)} & \text { otherwise. }\end{cases}
$$

